been newly started and opted for SC administration. Between October and December 2020, all suitable patients attending our infusion centre for vedolizumab were offered the option to switch to SC. Initially, the aim was to offer a SC dose to patients in place of their IV infusion with injection training by IBD specialists. This proved to be a challenge as it left a narrow window of time for homecare deliveries to be arranged for subsequent doses. Therefore, the remaining patients who agreed to the switch received an IV infusion at their baseline review, with the aim of administering the first SC dose in place of the next scheduled IV dose.

Outcomes include reasons for consenting or declining to switch, patient experience with using SC injections and time saved by not needing to travel to the infusion centre. Data on factors associated with poor outcomes from SARS-CoV-2 infection were collected, including co-morbidities, smoking status, concomitant medication and age.

Clinical baseline data collected as part of routine care included disease activity (modified Harvey-Bradshaw Index or Simple Clinical Colitis Activity Index), biochemical results including C-reactive protein, albumin, haemoglobin and platelet count, faecal calprotectin and quality of life using IBDControl. Trough vedolizumab levels were measured in patients who had had at least 3 IV doses previously. Patients will be reviewed after 12 weeks as part of the switching programme. Results 179 patients were offered the opportunity to change to SC vedolizumab $(54.2 \% \mathrm{CD}, 44.1 \% \mathrm{UC}, 1.7 \% \mathrm{IBDU})$, of which 125 (70\%) (64 (51.2\%) CD, 58 (46.4\%) UC and 3 (2.4\%) IBDU) agreed to the switch. The mean age (SD) was 55 (19.4). 11 patients were new to vedolizumab or reloading. The median time taken by patients (leaving home to returning home) to receive their infusions was 180 minutes (IQR 45 to $360)$.

The main reasons for agreeing to switch were patient preference to manage their treatment at home (70.4\%), concerns about contracting an infection at the infusion centre (15.7\%) and difficulty attending the infusion centre (15.7\%). Reasons for patients declining included not wanting to self-inject (28.3\%), needle phobia (15.2\%), and current instability of symptoms (15.2\%). There have been no major adverse events to date.

Conclusions This is a description of a service evaluation design to monitor outcomes in patients who have consented to transition from IV to SC vedolizumab at one IBD tertiary referral centre.

\section{PMO-16 EFFICACY AND SAFETY OF ELECTIVE SWITCHING FROM INTRAVENOUS TO SUBCUTANEOUS INFLIXIMAB: A MULTI-CENTRE COHORT STUDY}

1Philip J Smith* 1'Daniel Storey, ${ }^{1}$ Belle Gregg 1' ${ }^{1}$ Andrew Kneebone, 'Tracy Rimmer, 'Stevena Burke, ${ }^{2}$ Wan Yi Teoh, ${ }^{2}$ Stephan Vazeille, ${ }^{3}$ Solange Serna, ${ }^{1}$ Ashley Bond, ${ }^{1}$ Alan Steel, ${ }^{1}$ Martyn Dibb, ${ }^{1}$ Paul Collins, ${ }^{1}$ Edmund Derbyshire, ${ }^{1}$ Keith Bodger, ${ }^{1}$ Christopher Probert, ${ }^{3}$ Ajay Verma, ${ }^{1}$ Sreedhar Subramanian. ${ }^{1}$ Department of Gastroenterology, Liverpool University Hospitals Foundation NHS Trust, Liverpool, UK, Liverpool, UK; ' 'Liverpool School of Medicine, University of Liverpool, Liverpool, UK; ${ }^{3}$ Department of Gastroenterology, Kettering General Hospital, Kettering, UK

\subsection{6/gutjnl-2021-BSG.155}

Introduction Intravenous (IV) infliximab is a well-established therapy for inflammatory bowel diseases (IBD) patients. A subcutaneous (SC) formulation of infliximab has recently been shown to be as effective as IV infliximab in a randomised trial but there are no real world data to support elective switching. We aimed to assess the effectiveness of an elective switching program from IV to SC infliximab.

Methods Patients on maintenance IV infliximab were included in this retrospective multi-centre cohort study across two sites. Disease activity was monitored serially with Harvey-Bradshaw Index (HBI) for Crohn's disease (CD) and simple clinical colitis activity index (SCCAI) for Ulcerative colitis (UC) at baseline, 3 and 6 months. Faecal calprotectin (FCP), C-reactive protein (CRP) and infliximab levels were recorded at the same time points. Patients on $5 \mathrm{mg} / \mathrm{kg}$ every 8 weeks were switched to SC infliximab $120 \mathrm{mg}$ every other week (EOW), followed by those on 6 and 4 weekly IV infliximab to either EOW or weekly SC infliximab. Inclusion and exclusion criteria for switching were agreed within a standard operating procedure. Data was analysed using SPSS for Windows and Microsoft Excel, were $\mathrm{p}<0.05$ being significant (ns, non-significant).

Results 172 patients (109 CD, 57 UC, 6 IBD-U) were switched to SC infliximab since April 2020 (97 male vs. 75 female; mean age 39.7 years; mean weight $78.6 \mathrm{~kg}) .57 .6 \%$ of patients $(n=99)$ were on concurrent immunomodulators (mean thioguanine level $=259 \mathrm{pmol} / 8 \times 10^{8} \mathrm{RBC}$ ). 152 patients were switched to EOW dosing, 20 to weekly dosing. From baseline to 6 months post switch, HBI, SCCAI, FCP and CRP remained stable ( $p>0.05$, ns), but infliximab levels significantly increased from baseline (baseline mean $=9.8 \mathrm{ug} / \mathrm{mL}$ vs. 3 month mean $=14.6 \mathrm{ug} / \mathrm{mL} ; \mathrm{p}<0.0001)$ and then levels remained high between 3 and 6 month follow up (6 month mean $=15.0 \mathrm{ug} / \mathrm{mL} ; \mathrm{p}>0.05$, ns). 8 patients $(4.65 \%)$ had selflimiting skin injection site reactions, 1 UC patient $(0.58 \%)$ required corticosteroids for a disease flare, and 2 patients $(1.16 \%)$ developed perianal symptoms requiring examination under anaesthesia (EUA). Overall 4 patients (2.36\%) discontinued SC treatment due to adverse events ( 2 perianal disease patients requiring EUA, 1 flaring UC patient requiring oral steroids and 1 patient with neurological symptoms) and 1 $(0.58 \%)$ planned discontinuation for a patient in the $3 \mathrm{rd}$ trimester of pregnancy.

Conclusions SC infliximab is effective at maintaining remission in IBD patients switched from IV to SC infliximab with no evidence of inferiority at 6 month follow up. There is a significant increase in infliximab levels from baseline to 3 months which is maintained to 6 months post switch. SC infliximab appears to be safe with low rates of injection site reaction or other serious adverse events.

\section{PMO-17 CLINICAL PREDICTORS OF VEDOLIZUMAB RESPONSE IN CROHN'S DISEASE}

${ }^{1}$ Hajeena Saravanapavan*, ${ }^{1}$ Gregory Sebepos-Rogers, ${ }^{1}$ Konstantinos Fragkos, ${ }^{1}$ John Barragry, ${ }^{2}$ Hui Fen Koo, ${ }^{2} \mathrm{Ho}$ Juen Ko, ${ }^{1}$ loanna Parisi, ${ }^{1}$ Edward Seward, ${ }^{1}$ Sara McCartney, ${ }^{1}$ Stuart Bloom, ${ }^{1}$ Shameer Mehta, ${ }^{1}$ Faroog Rahman. ${ }^{1}$ Dept. Gastroenterology, University College Hospital, London, UK; ${ }^{2}$ University College London Medical School, London, UK

\subsection{6/gutjnl-2021-BSG.156}

Introduction Vedolizumab (VDZ) is an $\alpha 4 \beta 7$ integrin antagonist licensed for treatment of moderate to severe Crohn's disease (CD). We provide long-term data on best predictors of clinical and corticosteroid-free response, remission and persistence from a tertiary IBD centre. 